\title{
Easily Integrable platform for the deployment of a Remote Laboratory for microcontrollers
}

\author{
J. García-Zubia, I. Angulo, U. \\ Hernandez \\ Faculty of Engineering \\ University of Deusto \\ Bilbao, Spain \\ zubia@eside.deusto.es
}

\author{
M. Castro, E. Sancristobal \\ IEECD - Spanish Open University \\ UNED \\ Spain
}

\author{
P. Orduña, J. Irurzun, J. Ruiz de \\ Garibay \\ DeustoTech - Tecnológico \\ Fundación Deusto \\ Bilbao, Spain
}

\begin{abstract}
Remote laboratories are the natural solution in order to perform real experimentation under e-learning tools. Nevertheless these tools are the result of the research developed by the universities to cover their own needs without having in consideration the deployment of this technology by other institutions. This paper presents a hw prototype for a Remote Lab for microcontrollers that tries to solve these problems contributing new possibilities from the commercial and professional point of view.
\end{abstract}

\section{Remote laboratory; e-learning platform; microcontrollers}

\section{INTRODUCTION}

The advantages offered by e-learning platforms have caused important changes in education. However certain technical studies require the use of laboratories where develop real experiments with a greater didactic capacity than simulators [1].

Nowadays, the use of Remote Laboratories in universities is extending widely. A Remote $\mathrm{Lab}$ is a combination of hardware and software which allows a student to complete lab assignments remotely, e.g. from his home, just as if she was present in a laboratory. The student remotely controls the devices in the laboratory through a web interface, and monitors the outputs resulted from her interaction through a WebCam, a data file or using other electronic measuring instruments like oscilloscope or multimeter.

\section{ACTUAL SCENARIO}

Since the end of the 20th century, Remote Labs fill a very active research field in e-learning, and during the last five years the number of universities working on new Remote Labs has been multiplied. However, most of these new solutions do not match a professional level.

The fact that very few projects have been exported to other universities is just a proof of this statement. The most remarkable examples of this would be the VISIR Project [2] (BTH-Sweden) and the iLabs (MIT - USA) [3]. Another proof is that there is no market of commercial Remote Laboratories, neither a company supporting them, except National Instruments (NI).
The technical reasons behind this lack of professional or commercial projects in remote experimentation are the deficiencies of the software and hardware underneath.

From the point of view of the software, many Remote Labs have been developed without matching even minimum requirements. For instance, in a research asking 10 researchers in remote experimentation (MIT, BTH, EPFL, etc.) what features were more important for the development of a Remote Lab, the preferred ones were those related with software aspects (security, cross-platform, supported web browsers, etc.) instead of those related with hardware aspects (frequency, accuracy, etc.).

This shortage can only be addressed by designing the Remote Laboratory focusing appropriately on the software design and technologies used -as in the WebLab-Deusto Project using Web 2.0 technologies [4] (U.Deusto, Spain)-, or designing the Remote Laboratory on top of a LMS (Moodle, .LRN, Sakai, etc.) that will provide many transversal aspects of any Remote Laboratory.

On the other side, from the hardware viewpoint, the biggest problem is that the experiment is not autonomous, so part of its basic functionality is tightly coupled to the software side. It is usually difficult to isolate the hardware from the software providing the experiment, so it is not possible to unstuck the hardware side of the project and deploy it on other platform (such as an LMS) easily.

The users of a Remote Lab are the teachers and the students, and also the university. So a Remote Lab must be focused on teachers and students. Usually the teachers' requirements are forgotten by the researchers (because they usually will be the teachers). The teachers need a tool easy to install, to use, to manage, etc., more or less as Moodle is for them. The question is, are the Remote Labs easy to deploy? The answer is no, attending to the lack of deployed or commercialized Remote Laboratories.

Imagine that a high school want to install a Remote Laboratory, is it possible? Not today

The authors would like to acknowledge to the Spanish Science and Innovation Ministry for the support in the project TIN2008-06083-C03/TSI "s-Labs - Integración de Servicios Abiertos para Laboratorios Remotos y Virtuales Distribuidos" 


\section{PREVIOUS APPROXIMATION}

Due to the scenario described in the previous paragraph, during 2008 the Faculty of Engineering of the University of Deusto developed a Remote Laboratory in order to achieve two targets: get a low cost and easy to install platform [5].

After analyzing the architectures adopted in most known Remote Laboratories we come to the conclusion that all of them were based on a robust server (or more of one in some cases) entrusted of carrying out the tasks of interaction with the experiment and with the user.

The software installed in the server, designed generally in proportion to the particular needs of the developer university, impedes the deployment of the Remote Laboratory in other infrastructures and increases the cost of the final system. Therefore it is logical to think that eliminating the server of the architecture obtains a solution easier to install and certainly more economic.

Advances experienced by 8 bits microcontrollers during the last years allows to have low cost microservers where implement the tasks carried out previously by the computers. The selected microcontroller was the PIC18F97J60 launched by Microchip $\AA$, which includes an Ethernet controller inside as well as an incredible performance and diversity of resources to be a microcontroller of 8 bits.

Specifically, the WebLab-DEUSTO-PIC V0.1 is based on a system that implements two PIC18F97J60 microcontrollers that play different roles: Experiment and Server. The first one will run the program uploaded by the student, whereas the second one, the microserver, will allow the client to interact with the experiment: Upload the file to test, passwords control, inputs/outputs, etc. The microcontroller entrusted to execute the program created by the student is programmed with a bootloader, that is able to allocate in the program memory and execute a hex file received by tftp protocol. ("Fig. 1")

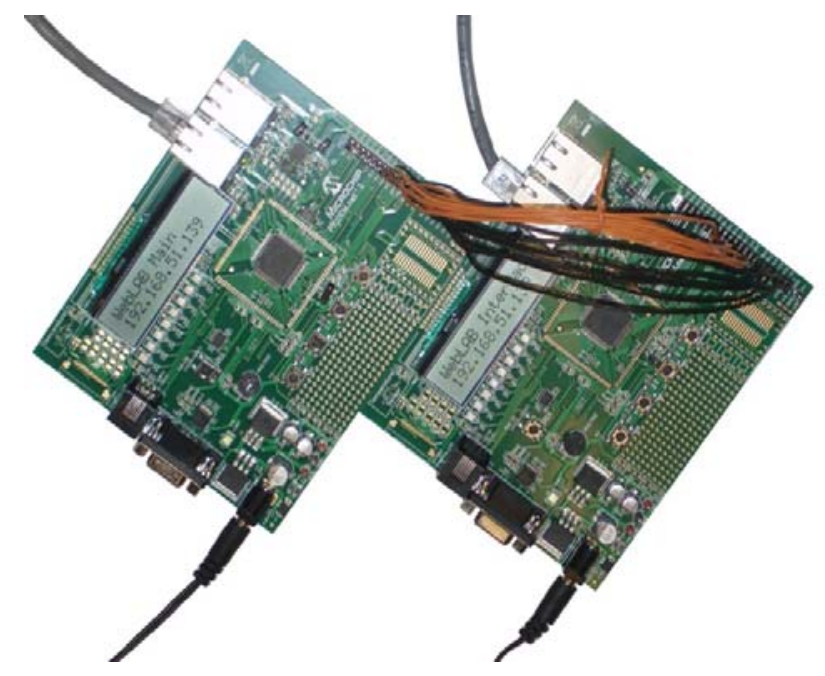

Figure 1. WebLab-DEUSTO-PIC v0.1 prototype
Student connects to the microserver using any web browser, log in with a password and once he is authorized he just can select the file that contains the program to test and upload it to the Remote Laboratory through a simple web page.

The file then is sent through simple tftp protocol to the microcontroller in charge of the experiment, which interrupts the execution of the previously loaded program when the reception of an UDP packet is detected, and the resident bootloader loads new hexadecimal file in the program memory of the microcontroller. Then new program is executed by the microcontroller until it is replaced with other one by the same or other student. The student that uploads a file is automatically redirected to other web page ("Fig. 2"), where he can experiment changing values of the inputs and monitoring the resultant outputs.

The employment of microservers contributes to the system two clear advantages:

- The cost of the complete system is lower than $200 €$.

- Being both cards, experiment and microserver compatible with the DHCP protocol, the installation of the system in any infrastructure is so simple as to power both cards and to connect them to the Ethernet network being accessible from moment the cards acquire IP addresses. This is what we name a Plug\&Play installation.

In the other hand, the limitations imposed by the microservers determine certain functional aspects of the Remote Laboratory:

- The limited performance of the selected microcontroller generates problems in moments of concurrent access.

- Tasks of management (administration of the users' queue, authentication, etc.) meet limited by the protocols included in the TCP/IP stack implemented by the microcontroller manufacturer.

- Being impossible to use a Webcam the monitoring of the outputs takes place across a web page losing realism in the development of the experiment.

- The resultant Remote Laboratory is not extensible to other laboratories further tan microcontrollers (CPLD, FPGA, etc.).

- It turns out to be very complicated to integrate the laboratory inside any e-learning platform. 


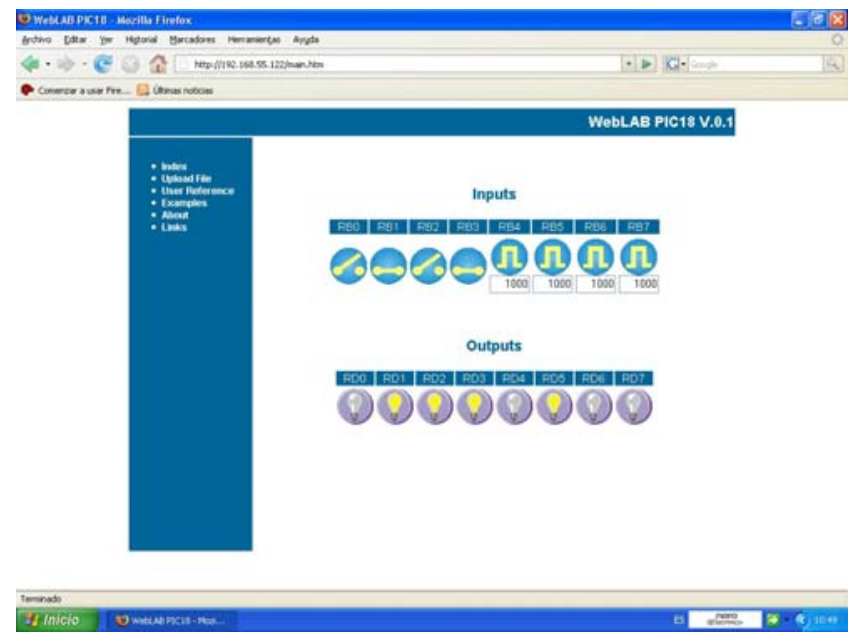

Figure 2. Web page for interacting with the experiment

\section{PROPOSED SOLUTION}

In the Faculty of Engineering of the University of Deusto play the role of users and developers of Remote Laboratories. This double point of view has allowed us to assure us on the one hand of the enormous didactic capacity of these tools as of the difficulty of its design and development as well as of the integration in an existing infrastructure like it can be the network and educative process of one university or school. This difficulty practically limits the use of Remote Laboratories to institutions with capacity for the research: universities. For this motive we have overturned our efforts in the design of a platform looking for a massive implantation in diverse educational centers on the basis of three fundaments:

- The Remote Laboratory must be a stable system. In order that the teacher considers the Remote Laboratory as any other didactic tool it must cause the minimal problems. In many occasions, the Remote Laboratories never pass from the state of prototype and the hardware is capable to external aspects like that a wire is disconnected because it hasn't a definitive connector or that the Webcam goes out of focus on not having been properly anchored. For these reasons the Remote Laboratory must have a professional appearance and the whole interaction between the teacher and the system must be to user's level making independent the tasks of developer and teacher completely. ("Fig. 3")

- The Remote Laboratory must be adaptable. The deployment of the system does not have to provoke a substantial change in the system of learning. It must adapt to the E-Learning platforms used by every educational center joining as one more resource inside it. Compatibility with Moodle, .LRN, Sakai or other learning platforms must be implemented.

- The Remote Laboratory must be extensible. Although it's been designed for teaching PIC microcontrollers, the architecture must support any other laboratory as other microcontrollers (Atmel, Freescale, etc.), DSP, CPLD, FPGA or any other programmable device.

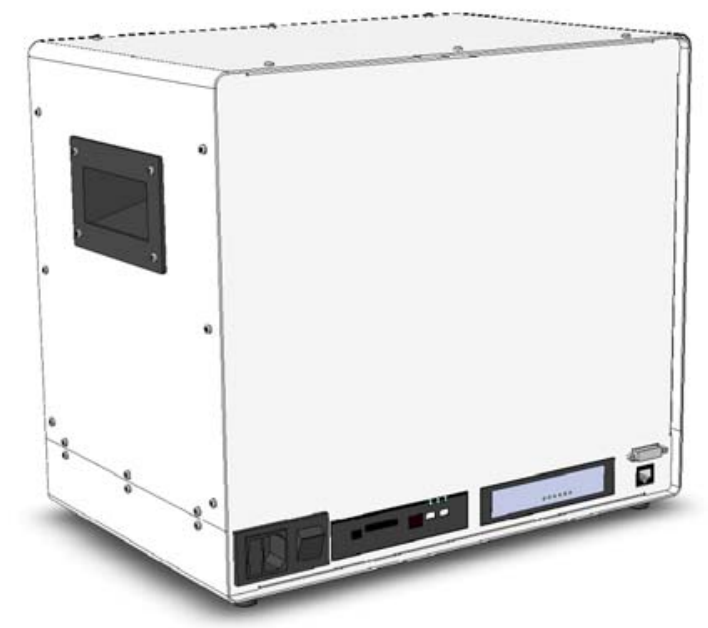

Figure 3. WebLAB-DEUSTO-PIC V2.0

\section{ARCHITECTURE OF WEBLAB-DEUSTO-PIC v2.0}

In order to achieve that the laboratory is extensible it has divided in the following parts: ("Fig. 4")

\section{A. Experiment board}

This board contains a microcontroller PIC18F97J60 on which the student will program the developed project. In this system the programming is carried out across the Ethernet network through a bootloader that receives the content to program in the memory of the microcontroller by tftp protocol. Being independent of the inputs and outputs it can be replaced for other including other device as a CPLD, a FPGA or p. e. an Atmel $\mu$ controller. Although it is convenient, in order to facilitate the assembling, it is not necessary to program the device through a TCP/IP protocol being possible to access to the USB ports of the server.

\section{B. Inputs board}

This board allows the student to manipulate the signals received by the experiment board. This board is also based in a PIC18F97J60 that receives from the interactive web page, through POST commands, the orders to set/reset digital inputs, change the value of analog inputs, receive serial data through UART port or any other kind of signal according to the nature of the experiment.

\section{Outputs board}

This board allows the student to watch, through the Internet camera, the outputs of the experiment. It can be designed customized for the experiment including most appropriate output peripherals. In WebLab-DEUSTO-PIC V2.0 it includes next elements:

- Two alphanumeric 2x16 LCD, one for experiment with the proper LCD and other one to visualize the information sent across the UART port.

- 8 LEDs directly connected to port B to manipulate digital outputs. 
- 8 LEDs connected through an $\mathrm{I}^{2} \mathrm{C}$ port expander (MCP23008) to experiment with Inter-Integrate Circuit Bus.

- 8 LEDs connected through a SPI port expander (MCP23S08) to experiment with Serial Peripheral Interface Bus.

- Futaba continuous rotation servo connected through a driver to experiment with PWM.

\section{DCS-5220 wireless motorized pan/tilt network camera}

Internet camera is placed over the outputs board allowing the student to watch the behavior of the experiment. Due to the size of the Outputs Card, it is convenient that the student can manage the camera focusing the part of the board involved in his own experiment. Interactive web page includes a control to move the camera.

\section{E. Fit-pc2 server}

The last advances in low format computers allow to arrange a powerful computer with small dimensions and low cost. New Intel Athom based computers make the employment of microservers slightly efficient being able to acquire robust servers, compatible with operating systems like Windows $7 \AA$ or Linux, that facilitate the development and the integration of the Remote Laboratory with similar dimensions and price. In WebLab-DEUSTO-PIC V2.0 a fit-pc2 ("Fig. 4") by Compulab has been incorporated to arrange all the management tasks needed by the experiment. Characteristics of this device are mentioned in "Table I". Functions to be done by this computer depend on the operational mode selected as is explained later.

TABLE I. FIT-PC2 SPECIFICATIONS

\begin{tabular}{|c|c|}
\hline \multicolumn{2}{|r|}{ Features } \\
\hline CPU & Intel Atom Z530 1.6GHz \\
\hline Chipset & Intel US15W SCH \\
\hline Memory & 1GB DDR2-533 on-board \\
\hline Storage & 160Gb $2.5 "$ SATA HDD \\
\hline Display & $\begin{array}{l}\text { Intel GMA500 graphics acceleration } \\
\text { DVI Digital output up to } 1920 \times 1200\end{array}$ \\
\hline Networking & $\begin{array}{l}1000 \text { BaseT Ethernet } \\
802.11 \mathrm{~g} \text { WLAN }\end{array}$ \\
\hline USB & 6 USB 2.0 High Speed ports \\
\hline IR & Programmable consumer IR receiver \\
\hline \multicolumn{2}{|r|}{ Mechanical and Environmental } \\
\hline Dimensions & $101 \times 115 \times 27 \mathrm{~mm}$ \\
\hline Case & $100 \%$ aluminum die cast body \\
\hline Weight & 370 grams / 13 ounces - including hard disk \\
\hline $\begin{array}{l}\text { Power } \\
\text { Consumptio } \\
\mathrm{n}\end{array}$ & $\begin{array}{c}6 \mathrm{~W} \text { at low CPU load } \\
<7 \mathrm{~W} \text { at } 1080 \mathrm{p} \mathrm{H.264} \mathrm{playback} \\
8 \mathrm{~W} \text { at full CPU load } \\
<1 \mathrm{~W} \text { at standby }\end{array}$ \\
\hline
\end{tabular}

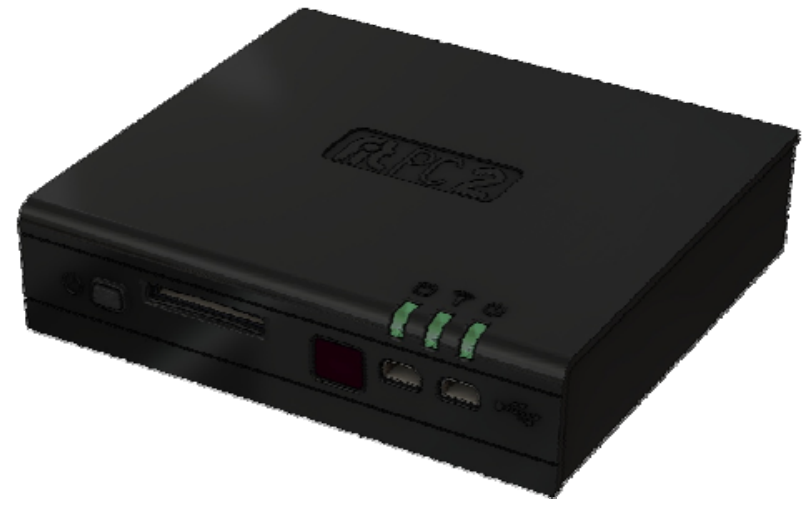

Figure 4. Low format computer "fit-pc2"

\section{F. 5 Port Fast Ethernet Switch}

To simplify the connection of all the elements with Ethernet connectivity to the network of the infrastructure the system has internally a Switch that interconnects them offering an only one RJ45 connector for its installation

\section{G. Power Supply}

25W Switching Power Supply, 5V/2,5A and 12V/1A Dual Output Voltage. Other output voltages and output models needed from other experiments are available with the same dimensions.

To decouple the hardware from the software allows to perform several experiments of different nature changing only two boards involved in the experiment: Experiment board, including one containing selected device and the logic to program it and Outputs board selecting the proper output peripherals for the experiment. ("Fig. 5")

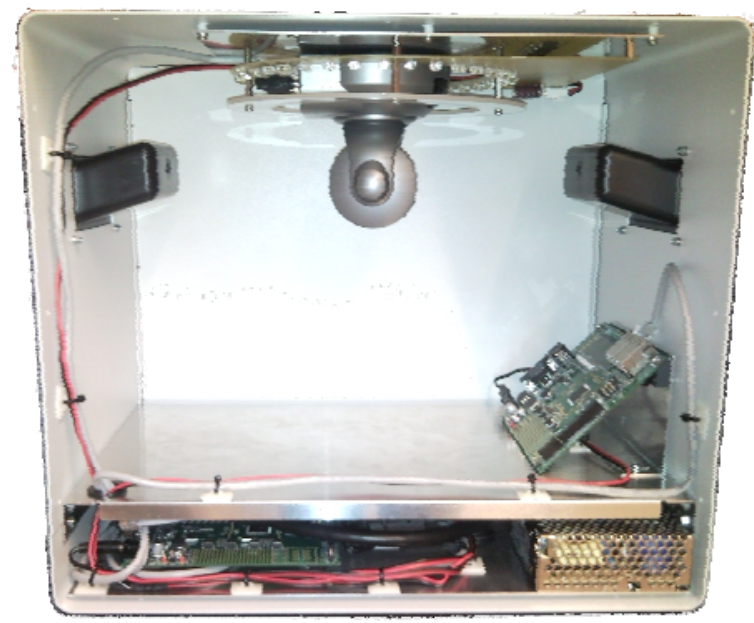

Figure 5. Transversal section of WebLAB-DEUSTO PIC V2.0 


\section{FUnCTIONAL CHARACTERISTICS OF WebLAB- DEUSTO-PIC V2.0}

To facilitate the deployment of this Remote Laboratory adjusting to the needs of any educational center the WebLabDEUSTO-PIC V2.0 can work according to 4 different operational modes:

- Plug\&Play Operational mode: This is the stand alone mode. None other element is required to perform the experiment. The inside computer realizes all administrative tasks: user authentication, user's queue management and $\log$ files generation so as the tasks related with the experiment: program the experiment board with the user hexadecimal file, serve the interactive web page and order to the inputs board all changes demanded by the user. "Fig. 6" shows a snapshot of the interactive web page working in this operational mode. Taking advantage of the high performance provided for fit-pc2 included WebLabDEUSTO-PIC V2.0 provides a completely autonomous Remote Laboratory with a high degree of integration on the part of the student.

- Integrated on WebLab-DEUSTO platform: The award-winning WebLab-Deusto project provides a web-based, experiment-agnostic, scalable software infrastructure which permits the University of Deusto to offer several laboratories to its students through the internet [6]. This platform is the result of years of research and provides perfect functionality for the development of remote experiments. The architecture is based in a central server in charge of all the management tasks and a microserver per experiment giving support to the communication with the specific hardware. Microserver task are implemented on the fitpc2 simplifying integration with the platform. Concurrent similar experiments are allowed reducing significantly waiting times of the students, one of the constraints in the use of Remote Laboratory in the same way that happens in on site ones. ("Fig. 7")

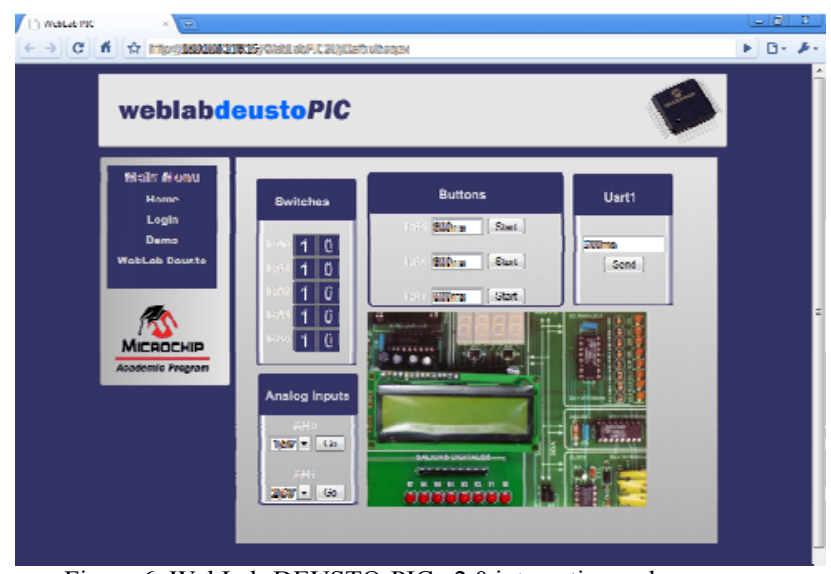

Figure 6. WebLab-DEUSTO-PIC v2.0 interactive web page.

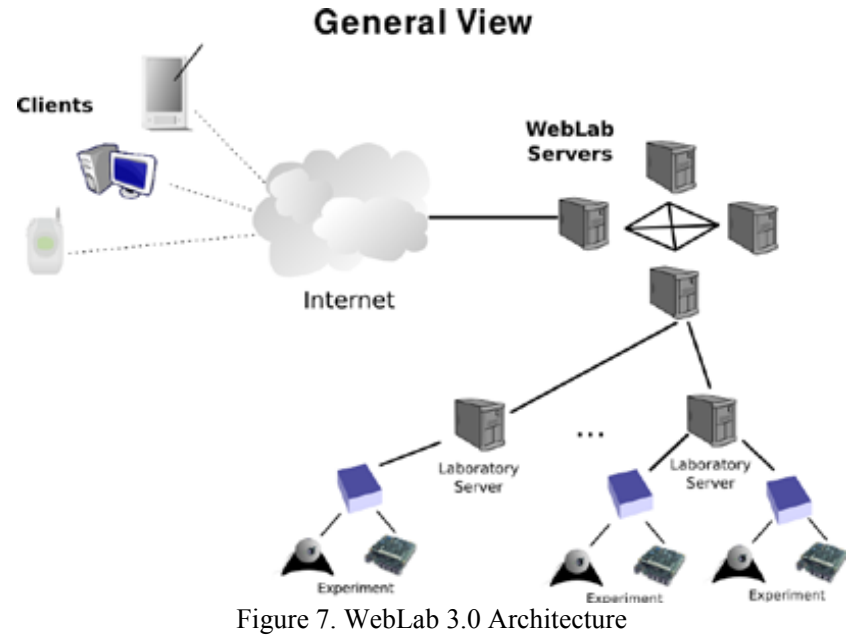

- Integrated in a learning platform: Employment of learning management systems (LMS) gains acceptance amongst educational institutions and their students. Integrate a Remote Laboratory on the institutional platform as one more resource offered improves the satisfaction obtained by the student simplifying the task of the teacher. WebLab-DEUSTO-PIC v2.0 is in process of being integrated on Moodle. A proper integration must allow include an experiment as if it were more available activity such as a questionnaire. Tasks of user authentication, storage of files uploaded by students and user's queue management must tu be performed by Moodle while the tasks of the experiment shall run on the fit-pc2. Due to the large number and diversity of learning platforms is difficult implement a solution for various systems, but the use of standards and the experience acquired with Moodle must simplify the integration on other LMS as .LRN or Sakai [7].

- Ready for xLAB integration: The University of Deusto is actively involved in developing the new platform for Remote Laboratories "xLab" in collaboration with the Massachusetts Institute of Technology (MIT) pretending that the WebLabDEUSTO-PIC v2.0 is one of the first laboratories offered under the same [8].

Regardless of the operational mode chosen, the hardware installation process is simple. All elements included: the outputs board, the inputs board, the experiment board, and internet camera support an announce protocol that provides access to their IP addresses in a simple manner. When the WebLab-DEUSTO-PIC v2.0 is plugged on the institutional network, the inside computer detects each device connected to the lab network and saves in one xml file the IP addresses assigned to each device. Simply configure the inside computer to access the full experiment. 
In addition in any operational mode the process of interaction is the same:

- The authorized student generates a binary file using preferred development environment (for example MPLAB IDE). The process of editing and assembling the program is intentionally removed from the laboratory allowing the student to use desired development tool and programming language in each moment

- Student connects to the Remote Lab waiting for his or her turn in case other students are using the lab. System manages user's queue trying to minimize waiting periods.

- When the student takes control of the lab via a web page selects the file to program the PIC microcontroller included in the experiment board. The file is sent through tftp protocol using the bootloader included preprogrammed in the microcontroller. The functionality of the bootloader is easy, after a reset the program waits 5 seconds to receive a binary file through tftp. If within that period, file is not received bootloader runs the last program stored, however if it receives a file compatible with the microcontroller, content is stored in program memory and bootloader launches the execution of the new program. The system should cause a reset in the experiment board before sending the file tftp. The reset is generated by the inputs board when receives a RESET POST command from the system.

- Automatically, after PIC is programmed system opens the interactive web page that allows the user to check the operation of the program. For 60 seconds (obviously timeout is configurable according with the experiment), the student can modify the value of the inputs from this web as seen on "Fig. 6" and monitor the result through the internet camera focusing the outputs board. Currently the interactive page allows control of 5 digital inputs whose value can be set or reset, three digital inputs that are activated during a period specified in milliseconds, two analog inputs whose value is selected through a list and allows the sending of a string through the asynchronous serial port (UART) by means of a text field.

- After this time, student is automatically asked to be relocated on the bottom of the user's queue.

\section{DEPLOYMENT OF THE WEBLAB-DEUSTO-PIC v2.0 ON A SUBJECT}

Since the development of Remote Laboratory WebLabDEUSTO-PIC v2.0 has been delayed until early November 2009 his employment in a real subject was not conducted until the second semester of course 2009-2010. The first course that will use this resource is "Digital Electronic Systems" belonging to the second year of the degree in Telecommunication
Engineering in the Faculty of Engineering, University of Deusto.

This experience will determine the satisfaction of the students and teacher as a result of using the system. The teacher of the selected course has not participated in developing the system beyond the requirements analysis.

Based on the results obtained in this pilot, from the next academic year WebLab- DEUSTO -PIC v2.0 will be deployed on other subjects of the same nature.

\section{CONCLUSIONS AND FUTURE}

Main contributions of the presented WebLab-DEUSTOPIC are focused in improving three fundamental characteristics in a remote laboratory: stability, adaptability and extensibility. The employment of the system in a real subject during the second semester of the course 2009-2010 will serve to measure the improvement with regard to other remote laboratories used previously.

In the near future work will be focussed on next objectives:

- Integrate the system in learning platforms type Moodle.

- Implement more units to study the improvement in student satisfaction having several similar laboratories concurrently with the consequent decrease in waiting times.

- Integrate new devices (DSP, CPLD, etc.) over the designed platform.

\section{REFERENCES}

[1] G. Alves, M. Gericota, J. Silva and J. Alves, "Large and small scale networks of Remote Labs: a survey", pp: 35-55, Advances on Remote Laboratories and e-learning experiences, Universidad de Deusto, Eds. J. García-Zubía, L. Gomes, ISBN 978-84-9830-077-2.

[2] I. Gustavsson, J. Zackrisson, L. Håkansson, I. Claesson, T. Lagö, “The VISIR project - an open source software initiative for distributed online laboratories," REV 2007, 2007, Porto

[3] Harward, V. J., del Alamo, J. A., Lerman, S. R., Bailey, P., Carpenter, J, "The iLab Shared Architecture: A Web Services Infrastructure to Build Communities of Internet Accessible Laboratories", Proceedings of the IEEE Vol. 96, No. 6, June 2008

[4] D. López-de-Ipiña, J. García-Zubia and P. Orduña, "Remote control of Web 2.0-enabled laboratories from mobile devices", e-Science 2006 , Dec. 4-6, 2006, Amsterdam, Netherlands

[5] J. García-Zubia, I. Angulo, U. Hernandez and P. Orduña, "Plug\&Play Remote Lab for microcontrollers: WebLab-DEUSTO-PIC", 7th European Workshop on Microelectronics Education May 28-30, 2008, BME - Budapest, Hungary.

[6] P. Orduña, J. García-Zubia, Jaime Irurzun, E. Sancristobal, S. Martín, M. Castro, D. López-de-Ipiña, U. Hernández, I. Angulo, J. M. González, "Designing experiment agnostic Remote Laboratories," REV 2009, 2225 June, Bridgeport

[7] Z. Bizonova, D. Ranc, M. Drozdova, "Model driven e-learning platform integration," in Proceedings of the EC-TEL 2007 PROLEARN Doctoral Consortium, Crete, Greece, September 18, 2007

[8] B. Cukalovic, "MIT integrated microelectronics device experimentation and simulation iLab," 2006, Massachusetts Institute of Technology. 


\section{AUTHORS}

J. García-Zubia is with the University of Deusto, Electronics and Automation Department, Avenida de las Universidades 24, 48007 Bilbao (Spain, he is Head of Dpt. Of Industrial Electronics, Control Engineering, and Computers Architecture of the Faculty of Engineering. He is the responsible of the Remote Lab at the University of Deusto (WebLab-DEUSTO: http://www.weblab.deusto.es). WebLab-Deusto has been implemented using web 2.0 techniques (AJAX, SOAP, etc), a novelty approach in Europe. Different works have been published explaining the results and the technology of this weblab and the evolution of WebLab-DEUSTO has been supported by different projects. (e-mail: zubia@deusto.es).

I. Angulo is with the University of Deusto in the Dpt. of Industrial Electronics, Control Engineering, and Computers Architecture of the Faculty of Engineering. He is the lead hardware designer and developer of WebLab-Deusto. (e-mail: iangulo@deusto.es).

P. Orduña, is a Computer Engineer by the University of Deusto, Bilbao 2007. Nowadays he is Research Assistant at the Ambient Intelligence department of DeustoTech - Tecnológico Fundación Deusto, and $\mathrm{PhD}$ student at the University of Deusto; his research is focused on Remote Laboratories. He is the lead software designer and developer of WebLab-Deusto. (e-mail: pablo.orduna@deusto.es).

M. Castro, Electrical and Computer Engineering educator in the Spanish University for Distance Education (UNED), has an industrial engineering degree from the ETSII (Industrial Engineering School) of the Madrid Polytechnic University (UPM) and a doctoral engineering degree from the same University. He received the Extraordinary Doctoral Award in the UPM and the Viesgo 1988 Award to the Doctoral Thesis improving the Scientific Research about the Industrial Process Electricity Application. He works as researcher, coordinator and director in different projects, ranging from solar system and advanced microprocessor system simulation to telematics and distance learning systems, acting now as and senior technical director. He is now with the UNED (Spanish University for Distance Education) as Professor in the Electronics Technology subject inside the Electrical and Computer Engineering Department as well as he is Director of the Department. (e-mail:mcastro@ieec.uned.es).

E. Sancristobal, San Cristobal Ruiz, Elio is a Computer Science Engineer by the Salamanca Pontifical University (UPS), Madrid 2002, has a Technical Engineering degree in computer networks (UPS), Madrid 1998. He finished his Doctoral Studies in Electronics Technology by the Electrical and Computer Engineering Department from the Spanish University for Distance Education (UNED), Madrid 2005. He worked for the University Distance Education Institute (IUED). Nowadays is working for the Computer Science Service Centre of the Spanish University for Distance Education (UNED) and is an associate professor Electrical and Computer Engineering Department from UNED. (e-mail: elio@ieec.uned.es).

J. Irurzun is a Computer Engineer by the University of Deusto, Bilbao 2009. He is Research Intern at the WebLab-Deusto Research Group and MSc student in Development and Integration of Software Solutions at the University of Deusto. (e-mail: irurzun@deusto.es).

U. Hernández is with the University of Deusto in the Telecommunications Department at the Faculty of Engineering. He is developer of the research group on web-based laboratories and he is in charge of the Remote Labs based on instruments control. He is involved too in the deployment in University of Deusto of the VISIR project leaded by the Blekinge Institute of Technology (Ronneby, Sweeden). (e-mail: unai.hernandez@deusto.es).

J. Ruiz-de-Garibay, is a Computer Engineer by the University of Deusto, Bilbao 2003. Nowadays he is Research Associate at the Ambient Intelligence department of DeustoTech - Tecnológico Fundación Deusto, and PhD student at the University of Deusto; his research is focused on Remote Laboratories based on Robotics. (email: jonathan.garibay@deusto.es). 
